in patients with multiple fistulas or previous repair surgeries. The length of follow-up, however, might have been insufficient to detect all complications. It is also possible that, while tunneled TVP is a good choice for patients who have undergone previous repair surgery and in whom tissue suitable for use might be difficult to find, simpler procedures might be more appropriate for primary repair attempts.

Original article Routh JC et al. (2006) Tunneled tunica vaginalis flap is an effective technique for recurrent urethrocutaneous fistulas following tubularized incised plate urethroplasty. J Urol 176: 1578-1581

\section{Dapoxetine safely delays premature ejaculation}

Dapoxetine is the first selective serotonin reuptake inhibitor (SSRI) specifically developed to treat premature ejaculation. Several SSRIs used to treat psychiatric disorders have been prescribed off-label for premature ejaculation, as SSRIs are known to delay ejaculation. Uniquely, dapoxetine has a very short half-life of $1.2 \mathrm{~h}$, which makes it suitable for on-demand use.

Pryor et al. have reported on two parallel, phase III, multicenter, randomized, doubleblind, placebo-controlled trials. They assessed 2,614 men with premature ejaculation (mean age 40.5 years); 870 received placebo, 874 received low-dose dapoxetine $(30 \mathrm{mg})$, and 870 received high-dose dapoxetine $(60 \mathrm{mg}$; 672,676 , and 610 men completed the study, respectively). One dose of dapoxetine was taken per $24 \mathrm{~h}$ period, 1-3 $\mathrm{h}$ before anticipated sexual intercourse. Intravaginal ejaculatory latency time (IELT) was assessed at baseline and at 4,8 , and 12 weeks.

Mean IELT at baseline for all groups was $\sim 0.91 \mathrm{~min}$, whereas mean IELTs at study end were $1.75 \mathrm{~min}, 2.78 \mathrm{~min}$, and $3.32 \mathrm{~min}$ for men receiving placebo, low-dose dapoxetine, and high-dose dapoxetine, respectively. IELT was significantly longer for dapoxetine-treated men than for placebo-treated men, and was also longer for men who received highdose dapoxetine than for men who received low-dose dapoxetine (both $P<0.0001$ ). Dapoxetine was effective from the first dose. Mild dizziness, nausea and syncope were described, but no severe adverse effects were reported.
Dapoxetine can delay ejaculation when given on demand, with the greatest IELT increases seen in men with the lowest baseline values.

Original article Pryor JL et al. (2006) Efficacy and tolerability of dapoxetine in treatment of premature ejaculation: an integrated analysis of two double-blind, randomised controlled trials. Lancet 368: 929-937

\section{Radical nephrectomy increases risk of new-onset chronic kidney disease}

A retrospective study of data from patients who underwent either partial or radical nephrectomy at the Memorial Sloan-Kettering Cancer Center, between July 1989 and September 2005 , has shown that radical nephrectomy is an important risk factor for new-onset chronic kidney disease (CKD), a condition associated with impaired survival and quality of life.

Huang et al. identified 662 patients who had undergone partial or radical nephrectomy for a solitary renal cortical tumor $\leq 4 \mathrm{~cm}$. All patients had pretreatment serum creatinine levels $\leq 124 \mu \mathrm{mol} / \mathrm{l}$, and a normal contralateral kidney. CKD was defined by a conventional glomerular filtration rate (GFR) threshold of $<60 \mathrm{ml} / \mathrm{min}$ per $1.73 \mathrm{~m}^{2}$, and by a more stringent threshold of $<45 \mathrm{ml} / \mathrm{min}$ per $1.73 \mathrm{~m}^{2}$. Surprisingly, the cohort had poorer baseline kidney function than expected: analysis revealed that $26 \%$ of the patients had preexisting CKD (using the conventional GFR threshold) before surgery.

After partial nephrectomy, the 3-year probability of freedom from new-onset CKD was $80 \%$ with the conventional GFR threshold, and $95 \%$ with the more stringent threshold. After radical nephrectomy, the 3-year probability of freedom from new-onset CKD was 35\% with the conventional GFR threshold, and $64 \%$ with the more stringent threshold.

Huang et al. say that radical nephrectomy is overused for patients with renal cortical tumors $\leq 4 \mathrm{~cm}$ in size. They recommend that an accurate, preoperative assessment of GFR should be an essential part of surgical decision-making for patients with these tumors.

Original article Huang WC et al. (2006) Chronic kidney disease after nephrectomy in patients with renal cortical tumours: a retrospective cohort study. Lancet Oncol 7: $735-740$ 